\title{
Quantum Brownian particle and memory effects
}

\author{
J. R. Brinati \\ Instituto de Física, Universidade Federal do Rio de Janeiro, Caixa Postal 68.528, 21945 Rio de Janeiro, Rio de Janeiro, Brazil \\ S. S. Mizrahi \\ Departamento de Física, Universidade Federal de São Carlos, Rodovia Washington Luiz Km 235, São Carlos, São Paulo, Brazil \\ B. M. Pimentel \\ Instituto de Física Teórica, Universidade Estadual Paulista, Rua Pamplona 145, 01405 São Paulo, São Paulo, Brazil
}

(Received 15 March 1991)

\begin{abstract}
The quantum Brownian particle, immersed in a heat bath, is described by a statistical operator whose evolution is ruled by a generalized master equation (GME). The heat bath's degrees of freedom are considered to be either white-noise or colored-noise correlated, while the GME is considered under either the Markov or non-Markov approaches. The comparisons between these considerations are fully developed, and their physical meaning is discussed.
\end{abstract}

PACS number(s): 05.40. $+\mathrm{j}$

\section{INTRODUCTION}

In recent years great interest $[1-7]$ arose in considering non-Markovian processes in quantum systems interacting with a heat bath or reservoir, since memory effects happen to be important and cannot be disregarded in the socalled generalized master equation (GME), which describes the time evolution of some system of interest $\mathcal{S}$.

A paradigm of a system commonly studied is the quantum Brownian particle $(\mathcal{S})$, represented by a harmonic oscillator (HO) of frequency $\Omega_{0}$ and unit mass. Furthermore, the system $\mathcal{S}$ is considered to interact with a medium, the reservoir $\mathcal{R}$, constituted of a very large number of independent HO's of frequencies $\omega_{n}$. In principle, the total Hamiltonian of $\mathfrak{S}+\mathcal{R}$ is quite simple, but the determination of the dynamical evolution of $\mathcal{S}$ is almost impossible to solve exactly (and is not necessary) due to the complexity involved in manipulating a great number of coupled integro-differential equations. Therefore, adequate approximations are introduced in the formalism in order to attain solutions that make transparent the essential physical aspects of the time evolution of $\mathcal{S}$. Among the interesting aspects that emerge in this study, one cites the thermal relaxation process that occurs in three different approaches: the drastic Markov approximation (MA) or white-noise MA (WNMA), corresponding to zero correlation time between the reservoir operators; the weaker MA or colored-noise MA (CNMA), which considers a finite correlation time; and finally the more precise non-Markov (NM) approach or colored-noise NM (CNNM). These concepts will be precisely defined later. Another interesting aspect to be treated corresponds to the memory effects in the dynamical evolution of the Brownian particle energy and its position-momentum correlation. Unlike other authors [6], we choose to attribute the Markov or non-Markov nomenclature to the system of interest $\mathcal{S}$ and not to the reservoir, since this is more similar to the original concepts [8]; however, we distinguish between white-noise and colored-noise approaches in the treatment of the reservoir operator correlations.

Our treatment differs from that of Refs. [1] and [7] in the picture we work: specifically, we obtain a GME in $c$ numbers that is used to calculate the mean values, whereas they solve Langevin equations. Among the considerations in our treatment we cite the following. (1) The $\mathcal{S}+\mathscr{R}$ interaction is in the rotating-wave approximation (RWA) [9], meaning the absence of rapidly oscillating terms. It is worth noting that our treatment is equivalent to the so-called modified RWA of Ref. [1]. (2) The weak-coupling approach, introduced in the GME of Peiers [10], takes into account terms up to second order in the interaction between $\mathcal{S}$ and $\mathcal{R}$. Since we work with the hypothesis that the reservoir remains in a global thermal equilibrium at any time, then this approach is sufficient to ensure an irreversible evolution [11] of the system $\mathcal{S}$. It is worthwhile to observe that in the above sense the interaction is weak for $\mathcal{R}$, whereas the damping may be strong or weak for $\mathcal{S}$, as discussed in Ref. [7].

Under these conditions the dynamical behavior of the quantum Brownian particle becomes characterized by four parameters: the natural frequency $\Omega_{0}$, the damping constant $\gamma$, which by its turn depends on the squared coupling constant $\left|K_{n}\right|^{2}$, the memory correlation time $\tau_{c}$, and the reservoir temperature $T$.

The aim of the present work is to compare the CNMA, considered by Velasco et al. $[2,5,6]$, with the lessstringent CNNM approach. Moreover, besides the usual $[1,2,5-7]$ memory kernel $e^{-|t| / \tau_{c}}$, which corresponds to a Cauchy distribution for the bath frequency spectrum, we also consider the memory kernel $\sin \left(t / \tau_{c}\right) / t$, corresponding to a square distribution, which has not been considered in those references.

The paper is organized as follows. In Sec. II the generalized master equation is introduced. In Sec. III the Hamiltonian is constructed, and in Sec. IV we define the 
HO frequency distributions of the reservoir to be used. Finally, Sec. V is devoted to the results and discussions.

\section{THE GENERALIZED MASTER EQUATION}

The exact Liouville equation for $\mathscr{S}+\mathcal{R}$, in the interaction picture, is given by

$$
\begin{aligned}
i \frac{\partial \hat{\rho}_{\mathcal{g}}(t)}{\partial t}= & {\left[\hat{V}_{\mathcal{J}}(t), \hat{\rho}_{\mathcal{I}}(0)\right] } \\
& -i \int_{0}^{t} d t^{\prime}\left[\hat{V}_{\mathcal{J}}(t),\left[\hat{V}_{\mathcal{g}}\left(t^{\prime}\right), \hat{\rho}_{\mathcal{g}}\left(t^{\prime}\right)\right]\right],
\end{aligned}
$$

where we assume a factorized form for the initial state $\hat{\rho}(0)=\hat{\rho}_{\delta^{\prime}}(0) \otimes \hat{\rho}_{\mathcal{R}}(T)$ and consider the reservoir statistical operator $\hat{\rho}_{\mathcal{R}}(T)$ representing an equilibrium canonical ensemble. Now, in order to get a master equation for the reduced statistical operator, we take the trace over the reservoir variables and introduce the basic irreversibility hypothesis [11] $\hat{\rho}_{\mathcal{I}}(t)=\hat{\rho}_{\mathscr{S} \mathcal{I}}(t) \otimes \hat{\rho}_{\mathcal{R}}(T)$. Then

$$
\frac{\partial \hat{\rho}_{\mathcal{I}}(t)}{\partial t}=-\int_{0}^{t} d t^{\prime} \hat{K}\left(t-t^{\prime}\right) \hat{\rho}_{\mathcal{I}}\left(t^{\prime}\right),
$$

where the memory kernel

$$
\hat{K}\left(t-t^{\prime}\right)=\operatorname{Tr}_{\mathcal{R}}\left(\hat{L}_{\mathrm{int}} e^{-i\left(t-t^{\prime}\right) \hat{L}_{0}} \hat{L}_{\mathrm{int}} \hat{\rho}_{\mathcal{R}}\right)
$$

depends on the Liouville operators

$$
\begin{aligned}
& \hat{L}_{\mathcal{S} \cdot}=\left[\hat{H}_{\mathcal{S}}, \cdot\right], \\
& \hat{L}_{\mathcal{R} \cdot}=\left[\hat{H}_{\mathcal{R}}, \cdot\right], \\
& \hat{L}_{\text {int }}=[\hat{V}, \cdot],
\end{aligned}
$$

with $\hat{L}_{0}=\hat{L}_{\mathcal{S}}+\hat{L}_{\mathscr{R}} ; \hat{H}_{\mathscr{S}}, \hat{H}_{\mathcal{R}}$, and $\hat{V}$ are, respectively, the system, the thermal bath, and the interaction Hamiltonian.

This approach is equivalent to the Born approximation for the exact kernel of the GME obtained by Peiers [10] according to the Zwanzig projection technique [12],

$$
\left\langle\hat{L}_{\text {int }} e^{-i\left(t-t^{\prime}\right)\left[\hat{L}_{0}+(1-\hat{P}) \hat{L}_{\text {int }}\right]} L_{\text {int }}\right\rangle_{R}+\hat{K}\left(t-t^{\prime}\right)+O\left(\left|K_{n}\right|^{4}\right) \text {, }
$$

where $\left|K_{n}\right|$ is the modulus of the coupling constant defined in Eq. (8).

In treating the integro-differential equation (2) there are cases that permit different approaches to it. For instance, the thermal-bath $\mathrm{HO}$ frequencies can be considered as a density distribution that may be constant for any frequency (white noise) or with some structure (colored noise). On the other hand, the system $\mathcal{S}$ can be considered as being local in time [substituting in Eq. (2) $\hat{\rho}_{\mathscr{S} \mathcal{I}}\left(t^{\prime}\right)$ by $\left.\hat{\rho}_{\mathfrak{S} \mathcal{g}}(t)\right]$, the Markov approximation, or can keep the nonlocal character already present in Eq. (2), the non-Markov approach. In what follows our aim is to make a quantitative as well as a qualitative comparison between the cases below.

(a) White noise for the thermal bath, which implies the Markov approximation, consists of assuming in Eq. (2) that the kernel $\hat{K}\left(t-t^{\prime}\right)=\delta\left(t-t^{\prime}\right) \hat{K}$. The $\delta$ function means that the reservoir spectrum is flat for any frequen- cy, so that the present behavior of $\mathcal{S}$ does not depend on the past history, since Eq. (2) becomes a linear differential equation with constant parameters.

(b) Colored noise for the thermal bath and $\hat{\rho}_{\mathscr{S} g}(t)$ local in time or Markovian (CNMA) consists of keeping the actual $\hat{K}\left(t-t^{\prime}\right)$ as a memory kernel while taking off $\hat{\rho}_{\mathscr{S} \mathcal{I}}(t)$ from the integral, so that Eq. (2) becomes a linear differential equation in $\hat{\rho}_{\mathscr{S} g}(t)$, but with time-dependent parameters.

(c) Colored noise for the thermal bath and $\hat{\rho}_{\mathcal{S g}}(t)$ nonlocal in time or non-Markovian (CNNM) keeps Eq. (2) without any approximation involving the time.

\section{THE MODEL HAMILTONIAN}

We consider one specific harmonic oscillator, the quantum Brownian particle $(\mathcal{S})$, interacting with a heat bath $(\mathscr{R})$, represented by a very large number of HO's in equilibrium at some temperature $T$. The model Hamiltonian is

$$
\hat{H}=\hat{H}_{\mathscr{S}}+\hat{H}_{\mathcal{R}}+\hat{V},
$$

where

$$
\hat{H}_{\mathcal{S}}=\Omega_{0} A^{\dagger} A
$$

and

$$
\hat{H}_{\mathcal{R}}=\sum_{n} \omega_{n} b_{n}^{\dagger} b_{n}
$$

are the system and reservoir Hamiltonians, respectively, and $\Omega_{0}$ and $\omega_{n}$ are the oscillator frequencies. The interaction between system and reservoir is considered in the RWA, namely,

$$
\hat{V}=\sum_{n}\left(K_{n}^{*} b_{n}^{\dagger} A+K_{n} b_{n} A^{\dagger}\right) \text {, }
$$

where $K_{n}$ and $K_{n}^{*}$ are coupling constants. In the above equations we considered that $\hbar=1$ and the operator commutation rules are bosonic type.

Substituting the Hamiltonian (5) into the GME (2), we arrive at the following equation:

$$
\begin{aligned}
\frac{\partial \hat{\rho}_{\mathcal{I}}(t)}{\partial t}=-\int_{0}^{t} d t^{\prime}\left(\left\{e^{-i \Omega_{0}\left(t-t^{\prime}\right)} \xi_{12}\left(t-t^{\prime}\right)\right.\right. \\
\times\left[A, A^{\dagger} \rho_{\mathcal{S} \mathfrak{I}}\left(t^{\prime}\right)\right] \\
+e^{i \Omega_{0}\left(t-t^{\prime}\right)} \xi_{21}\left(t-t^{\prime}\right) \\
\\
\left.\left.\quad \times\left[A^{\dagger}, A \rho_{\mathcal{S}}\left(t^{\prime}\right)\right]\right\}+ \text { H.c. }\right),
\end{aligned}
$$

where

$$
\begin{aligned}
& \xi_{12}\left(t-t^{\prime}\right)=\sum_{m=1}^{\infty}\left|K\left(\omega_{m}\right)\right|^{2} n\left(\beta \omega_{m}\right) e^{i \omega_{m}\left(t-t^{\prime}\right)} \\
& \xi_{21}\left(t-t^{\prime}\right)=\sum_{m=1}^{\infty}\left|K\left(\omega_{m}\right)\right|^{2}\left[1+n\left(\beta \omega_{m}\right)\right] e^{-i \omega_{m}\left(t-t^{\prime}\right)}
\end{aligned}
$$

are $c$-number kernel functions and 


$$
n\left(\beta \omega_{m}\right)=\left\langle b_{m}^{\dagger} b_{m}\right\rangle=\left(e^{\beta \omega_{m}}-1\right)^{-1}
$$

stands for the quanta mean value of the $m$ th $\mathrm{HO}$ and $\beta=\left(k_{B} T\right)^{-1} ; k_{B}$ is the Boltzmann constant. The functions $\xi_{12}$ and $\xi_{21}$ are correlation functions of the reservoir operators computed at different times.

Now we seek expressions for the mean values of quantities of interest belonging to $\mathcal{S}$, namely, $\langle A\rangle,\left\langle A^{\dagger} A\right\rangle$, $\left\langle A^{2}\right\rangle$, and their Hermitian conjugated. These quantities are essential to calculate the evolution in time of the energy, position, momentum, and $p-q$ correlation in the cases we mentioned in the preceding section. An adequate way to manipulate Eq. (9) is to work with the coherent-state labels as a mapping from a $q$-number to a $c$-number picture, namely,

$$
\begin{aligned}
& A \rightarrow \alpha, \\
& A^{\dagger} \rightarrow \alpha^{*}, \\
& \hat{\rho}_{\mathcal{S}}(t) \rightarrow P_{\mathcal{S I}}\left(\alpha, \alpha^{*} ; t\right) ;
\end{aligned}
$$

this mapping leads us to the $c$-number GME

$$
\begin{aligned}
\frac{\partial P_{\mathcal{S} \mathcal{I}}(t)}{\partial t}=\int_{0}^{t} d t^{\prime} & {[} \\
& +\Lambda_{2}\left(t-t^{\prime}\right) \frac{\partial}{\partial \alpha}\left[\alpha P_{\mathcal{S I}}\left(t^{\prime}\right)\right] \\
& + \text { c.c. }],
\end{aligned}
$$

where the kernel functions are now

$$
\begin{aligned}
\Lambda_{1}\left(t-t^{\prime}\right) & =\left[\xi_{21}\left(t-t^{\prime}\right)-\xi_{12}^{*}\left(t-t^{\prime}\right)\right] e^{i \Omega_{0}\left(t-t^{\prime}\right)} \\
& =\sum_{m}\left|K\left(\omega_{m}\right)\right|^{2} e^{-i\left(\omega_{m}-\Omega_{0}\right)\left(t-t^{\prime}\right)}
\end{aligned}
$$

and

$$
\begin{aligned}
\Lambda_{2}\left(t-t^{\prime}\right) & =\xi_{12}\left(t-t^{\prime}\right) e^{-i \Omega_{0}\left(t-t^{\prime}\right)} \\
& =\sum_{m}\left|K\left(\omega_{m}\right)\right|^{2} n\left(\beta \omega_{m}\right) e^{i\left(\omega_{m}-\Omega_{0}\right)\left(t-t^{\prime}\right)} .
\end{aligned}
$$

Then any mean value of a function involving $\alpha$ and $\alpha^{*}$ is calculated as a classical mean value; for instance,

$$
\left\langle f\left(\alpha, \alpha^{*}\right)\right\rangle_{t}=\int \frac{d^{2} \alpha}{\pi} P_{\mathcal{S}}\left(\alpha, \alpha^{*}, t\right) f\left(\alpha, \alpha^{*}\right) .
$$

\section{THE HO FREQUENCY DISTRIBUTIONS OF THE RESERVOIR}

In Eqs. (14) the discrete sums over the frequencies of the HO's are assumed to be closely spaced with a density distribution $g(\omega)$ such that one is permitted to substitute the sums by integrals,

$$
\sum_{m} \cdots \rightarrow \int_{0}^{\infty} d \omega g(\omega) \cdots
$$

and the kernel functions, Eqs. (14), become

$$
\begin{aligned}
& \Lambda_{1}\left(t-t^{\prime}\right)=\int_{0}^{\infty} d \omega g(\omega)|K(\omega)|^{2} e^{-i\left(\omega-\Omega_{0}\right)\left(t-t^{\prime}\right)} \\
& \Lambda_{2}\left(t-t^{\prime}\right)=\int_{0}^{\infty} d \omega g(\omega)|K(\omega)|^{2} n(\beta \omega) e^{i\left(\omega-\Omega_{0}\right)\left(t-t^{\prime}\right)}
\end{aligned}
$$

To characterize a colored noise, the function $g(\omega)$ is assumed to have some shape or structure distinct from the flat shape corresponding to the white noise, which characterizes a Markovian process of system $\mathcal{S}$.

Now, to calculate the mean value of some function $f\left(\alpha, \alpha^{*}\right)$ given by Eq. (15), one takes its Laplace transform, which results in an algebraic equation and thereafter turns back with the Mellin transform. For the above-mentioned cases we get the following Laplace transforms:

(a) $f\left(\alpha, \alpha^{*}\right)=\alpha$

Calling the Laplacian transform $\mathcal{L}_{t \rightarrow s}\left(\langle\alpha\rangle_{t}\right)=u(s)$, we obtain

$$
u(s)=\frac{u(0)}{s+\widetilde{\Lambda}_{1}(s)} .
$$

(b) $f\left(\alpha, \alpha^{*}\right)=\alpha^{2}$

Calling $\mathcal{L}_{t \rightarrow s}\left(\left\langle\alpha^{2}\right\rangle_{t}\right)=v(s)$, then

$$
v(s)=\frac{v(0)}{s+2 \widetilde{\Lambda}_{1}(s)} \text {. }
$$

(c) $f\left(\alpha, \alpha^{*}\right)=\alpha^{*} \alpha$

Calling $\mathcal{L}_{t \rightarrow s}\left(\left\langle\alpha^{*} \alpha\right\rangle_{t}\right)=w(s)$, then

$$
w(s)=\frac{s w(0)+2 \operatorname{Re} \widetilde{\Lambda}_{2}(s)}{s\left[s+2 \operatorname{Re} \widetilde{\Lambda}_{1}(s)\right]},
$$

where $\widetilde{\Lambda}_{1}(s)$ and $\widetilde{\Lambda}_{2}(s)$ are the Laplace transforms of $\Lambda_{1}\left(t-t^{\prime}\right)$ and $\Lambda_{2}\left(t-t^{\prime}\right)$, respectively.

For the above expressions $[(17 a)-(17 c)]$, the Mellin transform

$$
\left\langle f\left(\alpha, \alpha^{*}\right)\right\rangle_{t}=\frac{1}{2 \pi i} \int_{-i \infty+\epsilon}^{i \infty+\epsilon} d s e^{s t}\left\{\mathcal{L}_{t \rightarrow s}\left[\left\langle f\left(\alpha, \alpha^{*}\right)\right\rangle_{t}\right]\right\}
$$

is performed once the function $g(\omega)$ is defined beforehand.

Considering that $g(\omega)=g_{0}$ for all values of the frequencies, we obtain the WNMA, and the calculated mean values of the selected quantities mentioned in Sec. III are

$$
\begin{aligned}
& \langle A\rangle_{t}=\langle A\rangle_{0} e^{-\gamma t / 2-i \Omega_{0} t}, \\
& \left\langle A^{2}\right\rangle_{t}=\left\langle A^{2}\right\rangle_{0} e^{-\gamma t-2 i \Omega_{0} t}, \\
& \left\langle A^{\dagger} A\right\rangle_{t}=\left[\left\langle A^{\dagger} A\right\rangle_{0}-n\left(\beta \Omega_{0}\right)\right] e^{-\gamma t}+n\left(\beta \Omega_{0}\right),
\end{aligned}
$$

where $\gamma=2 \pi g_{0}\left|K\left(\Omega_{0}\right)\right|^{2}$ is the damping constant and \langle\rangle$_{0}$ stands for the initial mean values. In order to calculate the mean values of the Hermitian conjugated quantities of $A$ and $A^{2}$, it is sufficient to take the complex conjugate of $\langle A\rangle_{t}$ and $\left\langle A^{2}\right\rangle_{t}$.

In the following we are going to consider two specific 
shapes for $g(\omega)$ and compare the two approaches mentioned in Sec. II. In all the cases considered, the mean values of the selected quantities are calculated up to first order in $\tau_{c}$, the heat-bath correlation time, assumed to be much smaller than the system characteristic times.

\section{A. Square distribution}

The distribution function is considered to have a flat shape for a range of frequencies only, presenting a cutoff in the limits of its domain, i.e.,

$$
g(\omega)=\left\{\begin{array}{l}
g_{0}, \quad \Omega_{0}-\tau_{c}^{-1} \leq \omega \leq \Omega_{0}+\tau_{c}^{-1} \\
0 \text { otherwise }
\end{array}\right.
$$

with width $2 \tau_{c}^{-1}$.

For the CNMA we obtain

$$
\begin{aligned}
& \langle A\rangle_{t}=\langle A\rangle_{0}\left\{1+k\left[\chi_{c}(0)-\chi_{c}(\gamma t / k)\right]\right\} e^{-\gamma t / 2-i \Omega_{0} t}, \\
& \left\langle A^{2}\right\rangle_{t}=\left\langle A^{2}\right\rangle_{0}\left\{1+2 k\left[\chi_{c}(0)-\chi_{c}(\gamma t / k)\right]\right\} e^{-\gamma t /-2 i \Omega_{0} t},
\end{aligned}
$$

and

$$
\begin{aligned}
\left\langle A^{\dagger} A\right\rangle_{t}= & {\left[\left\langle A^{\dagger} A\right\rangle_{0}-n\left(\beta \Omega_{0}\right)\right] } \\
& \times\left\{1+2 k\left[\chi_{c}(0)-\chi_{c}(\gamma t / k)\right]\right\} e^{-\gamma t}+n\left(\beta \Omega_{0}\right),
\end{aligned}
$$

where $k=\gamma \tau_{c}$ is a factor that characterizes the memory effects in the time evolution of the mean values.

For the CNNM approach the mean values are

$$
\begin{aligned}
\langle A\rangle_{t}=\langle A\rangle_{0}[ & {\left[1+\frac{k}{\pi}(1-\gamma t / 2)\right] e^{-\gamma t / 2} } \\
& \left.-k \chi_{c}(\gamma t / k)\right] e^{-i \Omega_{0} t}, \\
\left\langle A^{2}\right\rangle_{t}=\left\langle A^{2}\right\rangle_{0} & {\left[\left[1+\frac{2 k}{\pi}(1-\gamma t)\right] e^{-\gamma t}\right.} \\
& \left.-2 k \chi_{c}(\gamma t / k)\right] e^{-2 i \Omega_{0} t},
\end{aligned}
$$

and

$$
\begin{aligned}
\left\langle A^{\dagger} A\right\rangle_{t}= & {\left[\left\langle A^{\dagger} A\right\rangle_{0}-n\left(\beta \Omega_{0}\right)\right] } \\
& \times\left[\left(1+\frac{2 k}{\pi}(1-\gamma t)\right] e^{-\gamma t}-2 k \chi_{c}(\gamma t / k)\right] \\
& +n\left(\beta \Omega_{0}\right) .
\end{aligned}
$$

In Eqs. (20) and (21), $\chi_{c}(y)$ is a function defined as

$$
\chi_{c}(y)=\frac{1}{\pi}[\cos (y)+y \operatorname{si}(y)] \text {. }
$$

\section{B. Cauchy distribution}

The Cauchy distribution function is a smooth continuous function for all values of frequencies, i.e.,

$$
g(\omega)=g_{0} \frac{1}{1+\left(\omega-\Omega_{0}\right)^{2} \tau_{c}^{2}},
$$

with a peak at $\omega=\Omega_{0}$. All the mean values are calculated, as in the previous case, up to first order in $\tau_{c}$, besides involving also a first order in the ratio $\gamma / \Omega_{0}$.

For the CNMA we get

$$
\begin{aligned}
\langle A\rangle_{t}=\langle A\rangle_{0}[ & +\frac{k}{2}[\sigma(t)-\sigma(0)] \\
& \left.+\frac{k}{2}\left(1-e^{-\gamma t / k}\right)\right] e^{-z \gamma t / 2-i \Omega_{0} t}, \\
\left\langle A^{2}\right\rangle_{t}=\left\langle A^{2}\right\rangle_{0}\{1+k[\sigma(t)-\sigma(0)] & \\
& \left.+k\left(1-e^{-\gamma t / k}\right)\right\} e^{-z \gamma t-2 i \Omega_{0} t},
\end{aligned}
$$

and

$$
\begin{aligned}
\left\langle A^{\dagger} A\right\rangle_{t}= & {\left[\left\langle A^{\dagger} A\right\rangle_{0}-n\left(\beta \Omega_{0}\right)\right] } \\
& \times\{1+k \operatorname{Re}[\sigma(t)-\sigma(0)] \\
& \left.\quad+k\left(1-e^{-\gamma t / k}\right)\right\} e^{-\gamma t}+n\left(\beta \Omega_{0}\right),
\end{aligned}
$$

where the integral

$$
\sigma(t)=-\frac{1}{\pi} \int_{\omega \tau_{c}}^{\infty} d x \frac{e^{i x t / \tau_{c}}}{x^{2}\left(1+x^{2}\right)}
$$

is calculated by numerical methods and

$$
z=1+\frac{i}{2 \pi} \ln \frac{\Omega_{0}^{2} \tau_{c}^{2}}{1+\Omega_{0}^{2} \tau_{c}^{2}},
$$

where $z \gamma$ is a pole that occurs in the Mellin transform, Eq. (18).

The CNNM approach gives

$$
\begin{aligned}
\langle A\rangle_{t}=\langle A\rangle_{0}\{ & {\left[1+\frac{k}{2}[1-\sigma(0)]\left[1-\frac{z \gamma t}{2}\right]\right] e^{-z \gamma t / 2} } \\
& \left.-\frac{k}{2} e^{-(1-k / 2) \gamma t / k}+\frac{k}{2} \sigma(t)\right\} e^{-i \Omega_{0} t}
\end{aligned}
$$

$$
\begin{array}{r}
\left\langle A^{2}\right\rangle_{t}=\left\langle A^{2}\right\rangle_{0}\left(\{1+k[1-\sigma(0)](1-z \gamma t)\} e^{-z \gamma t}\right. \\
\left.-k e^{-(1-k) \gamma t / k}+k \sigma(t)\right) e^{-2 i \Omega_{0} t},
\end{array}
$$

and

$$
\begin{aligned}
\left\langle A^{\dagger} A\right\rangle_{t}= & {\left[\left\langle A^{\dagger} A\right\rangle_{0}-n\left(\beta \Omega_{0}\right)\right] } \\
& \times\left(\{1+k[1-\operatorname{Re} \sigma(0)](1-\gamma t)\} e^{-\gamma t}\right. \\
& \left.\quad-k e^{-(1-k) \gamma t / k}+k \operatorname{Re} \sigma(t)\right)+n\left(\beta \Omega_{0}\right) .
\end{aligned}
$$




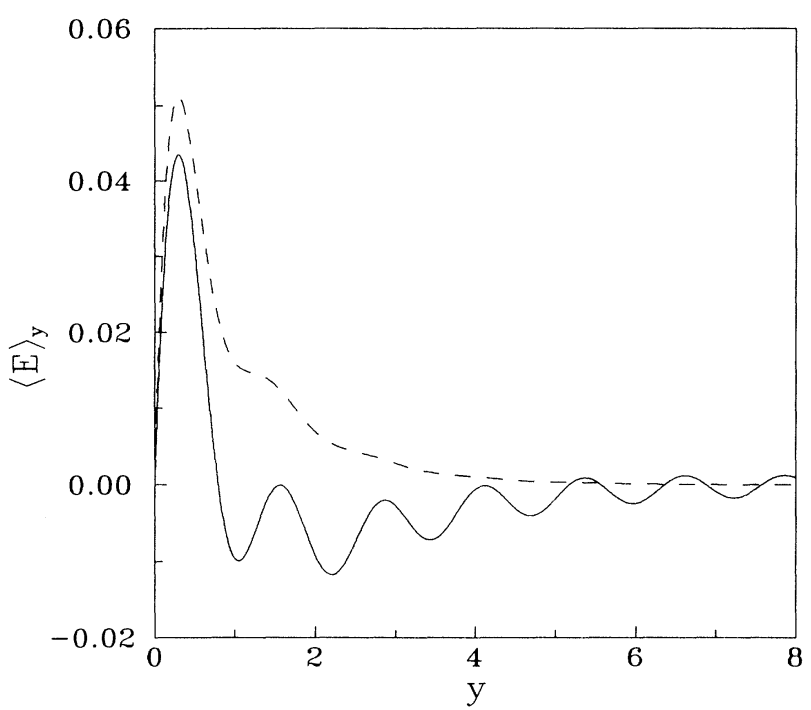

FIG. 1. Time evolution of the Brownian particle energy for the square distribution function, Eq. (20). The solid line corresponds to the CNNM approach and the dashed line corresponds to the CNMA. The independent variable $y=\gamma t$. In all these curves the WNMA was subtracted.

\section{RESULTS AND DISCUSSION}

To characterize the behavior of the quantum Brownian particle we calculate the dynamical evolution of the energy and the $p-q$ correlation. These quantities are related to the mean values $\langle A\rangle_{t},\left\langle A^{2}\right\rangle_{t}$, and $\left\langle A^{\dagger} A\right\rangle_{t}$, obtained in the preceding section, as follows:

$$
\langle E\rangle_{t}=\Omega_{0}\left(\left\langle A^{\dagger} A\right\rangle_{t}+\frac{1}{2}\right)
$$

and

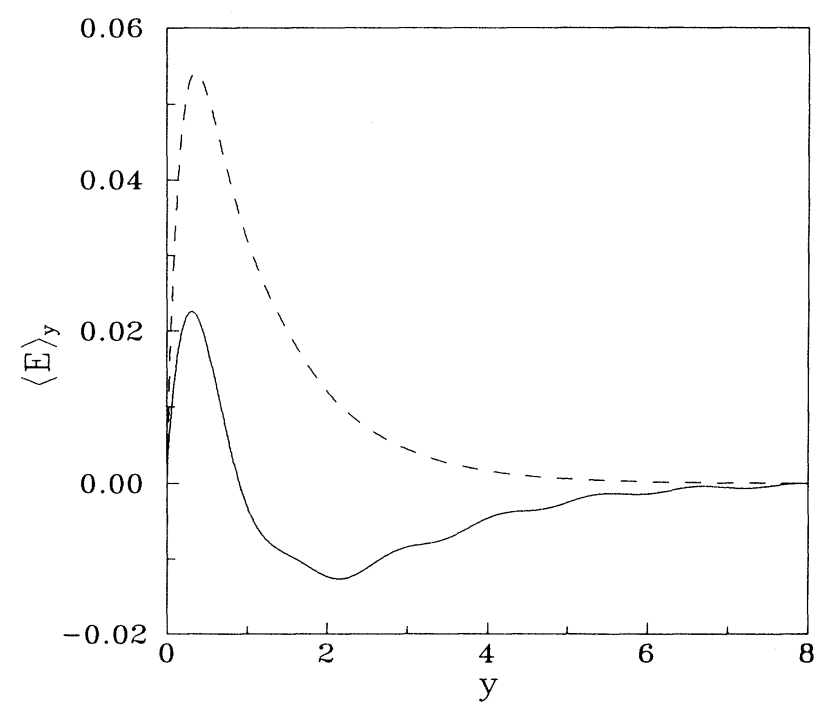

FIG. 2. Time evolution of the Brownian particle energy for the Cauchy distribution function, Eq. (24). All data are the same as in Fig. 1.

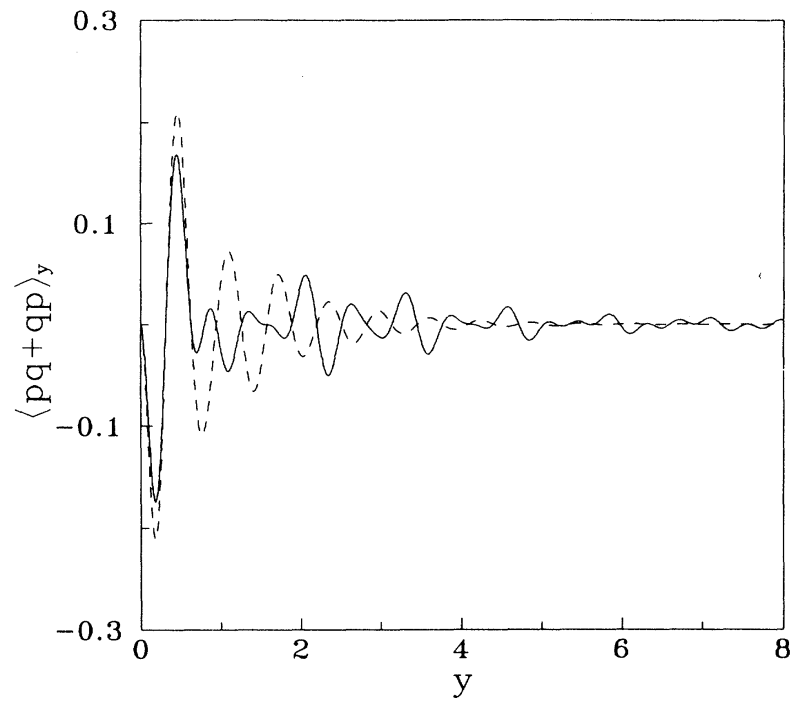

FIG. 3. Time evolution of the $p-q$ correlation for the square distribution function, Eq. (20). All data are the same as in Fig. 1.

$$
\langle p q+q p\rangle_{t}=i\left(\left\langle A^{\dagger 2}\right\rangle_{t}-\left\langle A^{2}\right\rangle_{t}\right),
$$

which present a characteristic transient behavior for each of the cases pointed out in Sec. II.

Figures 1 and 2 exhibit the energy versus time behavior for the square and Cauchy distributions, respectively. We consider the Glauber coherent states as the initial condition. The WNMA, considered as a background, was subtracted from the CNMA and CNNM expressions in order to stress their different behavior.

For the square spectrum case, Fig. 1, the CNNM ap-

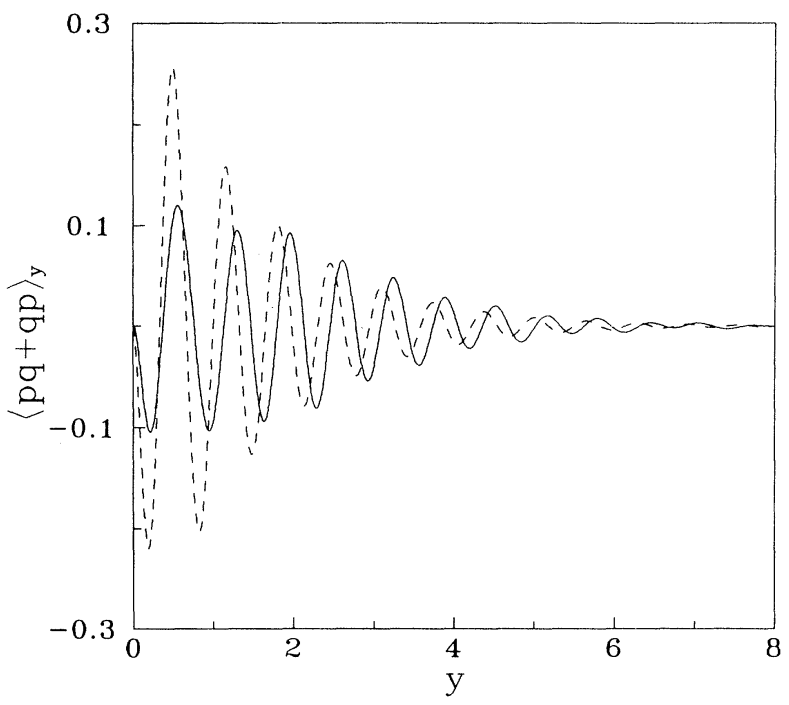

FIG. 4. Time evolution of the $p-q$ correlation for the Cauchy distribution function, Eq. (24). All data are the same as in Fig. 1. 
proach shows remarkable oscillations (solid line), even asymptotically, in comparison to the CNMA (dashed line), which presents no significant oscillatory structure. Figure 2 compares the energy versus time behavior for the Cauchy distribution. The CNNM curve presents a behavior similar to the square case, but now the oscillations are weaker, while the CNMA has a smooth structure. Asymptotically, all the curves of Figs. 1 and 2 go to zero, which corresponds to the thermal equilibrium energy.

The $p$ - $q$ correlations are depicted in Figs. 3 and 4 for the square and Cauchy distributions, respectively, and again we subtracted the WNMA from the CNMA and CNNM expressions. In both figures we observe an oscillatory behavior for the CNNM (solid line) and the CNMA (dashed line). For initial times, the oscillation amplitudes are more pronounced for the CNMA than for the CNNM approach, and this behavior changes as time increases. For the square distribution, Fig. 3, while the CNMA amplitudes decrease monotonically with time, the CNNM exhibits an exotic oscillatory behavior.

In all the calculations, to get the curves of these figures we used $n\left(\beta \Omega_{0}\right)=0.58,\left\langle A^{\dagger} A\right\rangle_{0}=1$, and $k=0.2$. For the Cauchy distribution we fixed the ratio $\gamma / \Omega_{0}=0.2$.

We can have a better qualitative insight of the different characteristics of the Markov approximation and nonMarkov approach when we consider the zero-time delay of the correlations

$$
\begin{aligned}
I(t) & =\left\langle A^{2}\right\rangle_{t}-\langle A\rangle_{t}^{2} \\
\text { and } & \\
\qquad J(t) & =\left\langle A^{\dagger} A\right\rangle_{t}-\left\langle A^{\dagger}\right\rangle_{t}\langle A\rangle_{t} .
\end{aligned}
$$

Concerning the Markov approximation and an initial coherent state, we can verify, from Sec. IV, that the quantity $I(t)$ is null, regardless of whether it is considered a white-noise or colored-noise bath (either square or Cauchy distributions). On the other hand, the strength of the correlation $J(t)$ depends essentially on the temperature through $n\left(\beta \Omega_{0}\right)$; specifically, when $T=0$, $J(t)=0$ at any time, meaning that an initial coherent state remains coherent as it evolves.

For the non-Markov approach and an initial coherent state we have a qualitatively different situation. The correlations $I(t)$ and $J(t)$ are time dependent, being proportional to the constant $k=\gamma \tau_{c}$; besides this, the strength of $J(t)$ also depends on $n\left(\beta \Omega_{0}\right)$ in a similar way as in the CNMA. So one verifies that even at $T=0$, an initial coherent state has its coherence destroyed as it begins to evolve. This fact is crucial to discern between a Markov and non-Markov treatment of a system under study.

\section{ACKNOWLEDGMENT}

This work was supported in part by Conselho Nacional do Desenvolvimento Científico e Tecnológico, Brazil.
[1] K. Lindenberg and B. J. West, Phys. Rev. A 30, 568 (1984).

[2] J. Bréton, A. Hardisson, F. Mauricio, and S. Velasco, Phys. Rev. A 30, 542 (1984).

[3] V. Capek and V. Szocs, Czech. J. Rev. B 36, 1182 (1986).

[4] J. R. Brinati, B. M. Pimentel, S. S. Mizrahi, and S. A. Carias de Oliveira, Can. J. Phys. 66, 1044 (1988).

[5] S. Velasco, J. A. White, and A. C. Hernandez, Phys. Rev. A 39, 3653 (1989).

[6] J. A. White and S. Velasco, Phys. Rev. A 40, 3156 (1989).

[7] F. Haake and R. Reibold, Phys. Rev. A 32, 2462 (1985); F.
Haake, in Statistical Treatment of Open Systems, edited by G. Höhler, Springer Tracts in Modern Physics Vol. 66 (Springer, Berlin, 1973).

[8] M. Lax, Phys. Rev. 129, 2342 (1963); 145, 109 (1966).

[9] W. H. Louisell, Quantum Statistical Properties of Radiation (Wiley, New York, 1973).

[10] W. Peiers, Physica 57, 565 (1972); 58, 229 (1972).

[11] A. S. Davydov, Quantum Mechanics, 2nd ed. (Pergamon, New York, 1976).

[12] R. W. Zwanzig, Lect. Theor. Phys. 3, 106 (1960). 